CYSTIC FIBROSIS

\title{
Neutrophil cell death, activation and bacterial infection in cystic fibrosis
}

\author{
A P Watt, J Courtney, J Moore, M Ennis, J S Elborn
}

Thorax 2005;60:659-664. doi: 10.1136/thx.2004.038240

See end of article for authors' affiliations

.....................

Correspondence to:

Dr A Watt, Department of Clinical Biochemistry and Metabolic Medicine,

Institute of Clinical Science,

The Queen's University of

Belfast, Grosvenor Road,

Belfast BT12 6BJ, UK;

a.watt@qub.ac.uk

Received

26 November 2004

Accepted 9 May 2005

\begin{abstract}
Background: Cystic fibrosis (CF) is characterised by chronic endobronchial bacterial infection and neutrophil mediated inflammation. Neutrophil apoptosis is essential for the resolution of inflammation. This study assessed the relationship between levels of neutrophil apoptosis and sputum microbiology in matched clinically stable patients with CF.

Methods: Sputum was induced from 34 patients (nine with no Gram negative infection, 10 colonised with Pseudomonas aeruginosa, 10 with Burkholderia cenocepacia, and five with other infections). Apoptotic neutrophils measured by flow cytometric Annexin $\mathrm{V} /$ propidium iodide staining and morphology were similar in all groups.

Results: Patients infected with $P$ aeruginosa or $B$ cenocepacia had a significantly lower percentage of viable neutrophils in the sputum than those with no Gram negative infection (Kruskal-Wallis $p=0.01$, median (interquartile range (IQR)) $14.2 \%(9.4-21.6), 15.8 \%(12.3-19.5)$, and $48.4 \%(23.0-66.4)$; $p=0.003$ and $p=0.002$, respectively). They also had significantly higher levels of secondary necrotic granulocytes in sputum than patients with no Gram negative infection (Kruskal-Wallis $p<0.0001$, median (IQR) 55.5\% (48.4-64.5), 50.4\% (44.6-61.9), and 24.8\% (14.4-30.5); $p<0.0001$ and $p<0.0001$, respectively). Neutrophils $\left(\times 10^{6} / \mathrm{g}\right.$ sputum) in $P$ aeruginosa infected patients (Kruskal-Wallis $p=0.05$, median (IQR) $6.3(3.5-12.7))$ and $B$ cenocepacia infected patients (5.7 (1.5-14.5)) were significantly higher than in the group with no Gram negative infection $(0.5(0.5-4.3), p=0.03$ and 0.04 , respectively). Conclusion: These results suggest that cell death and clearance may be altered in patients with $\mathrm{CF}$ colonised with $P$ aeruginosa and $B$ cenocepacia compared with those with no Gram negative infection.
\end{abstract}

$\mathrm{L}$ ung damage due to bacterial infection remains the main cause of morbidity and mortality in cystic fibrosis (CF). ${ }^{1}$ -The lungs of patients with CF become colonised in early infancy with micro-organisms such as Staphylococcus aureus and Haemophilus influenzae, which may damage epithelial surfaces, leading to increased attachment of and eventual replacement by Pseudomonas aeruginosa (PA). ${ }^{2}$ Once acquired, PA will invariably colonise the patient for life. The virulence and longevity of PA infections can be attributed to a number of factors including biofilm formation in the lung. ${ }^{1}$ In recent decades, Burkholderia cenocepacia (BC) (formerly B cepacia genomovar IIIa) has also been identified as an important lung pathogen eliciting a range of disease states from asymptomatic carriage to the lethal "cepacia syndrome". ${ }^{3}$ Colonisation with both these micro-organisms is detrimental to the patient; however, pulmonary colonisation by BC is observed to lead to an accelerated decline in lung function. The mechanism by which BC causes this deterioration is unclear and, although virulence factors have been identified, none appear to account for its pathogenicity. ${ }^{4-6}$

In the lungs, neutrophils are important in innate defence against infection, preventing spread of micro-organisms by phagocytosis and release of toxic agents such as proinflammatory cytokines, proteases, and reactive oxygen species. ${ }^{7}$ In a healthy lung inflammation resolves naturally and the tissue returns to normal. ${ }^{8}$ In CF, however, inflammation is characterised by a severe and sustained neutrophil mediated response. It has been hypothesised that this persistent inflammatory response and abnormal accumulation of cells may arise from failure or inefficiency of the mechanisms responsible for resolution of inflammation and restitution of tissue homeostasis-that is, apoptosis and subsequent clearance by macrophages. ${ }^{9}$ Apoptosis is a granulocyte clearance mechanism which limits tissue injury; in contrast to necrosis, granulocyte secretory processes are shut down and the intact senescent neutrophil is removed by macrophages using phagocytic recognition mechanisms and triggering release of anti-inflammatory mediators. ${ }^{9}$ Secondary necrosis of apoptotic cells is likely to occur if phagocytic clearance is disrupted resulting in amplification of inflammation. ${ }^{9}$ The apoptotic pathway is susceptible to modulation by cytokines, implying active control of cell death during the course of inflammation in vivo. ${ }^{10}$ Evidence also exists to suggest that pathogens may have the capability to modulate apoptosis. ${ }^{11-14}$ PA secretes a phenazine pigment exotoxin called pyocyanin which has pro-apoptotic effects on neutrophils in culture. ${ }^{12}$ Hemolysin, a toxin produced by BC, is also capable of inducing apoptosis and degranulation of mammalian phagocytes. ${ }^{13}$

It has previously been shown that, in CF patients who are clinically stable, levels of inflammatory markers are higher than in a matched non-CF population. ${ }^{15}$ There is not such a clear pattern between levels of inflammation and various different infecting organisms. In this study we have examined the relationship between neutrophil cell death, activation, inflammatory mediator levels, and sputum microbiology in matched clinically stable CF patients.

\section{METHODS}

\section{Patients}

Thirty four clinically stable patients with CF participated in the study. A diagnosis of CF was confirmed by a typical phenotype, genotype and/or sweat test. A cross sectional

Abbreviations: BC, Burkholderia cenocepacia; CF, cystic fibrosis; CRP, $\mathrm{C}$-reactive protein; $\mathrm{FEV}_{1}$, forced expiratory volume in 1 second; $\mathrm{FVC}$, forced vital capacity; NE, neutrophil elastase; PA, Pseudomonas aeruginosa; PS, phosphatidylserine 
Table 1 Median (interquartile range) demographic data of study patients

\begin{tabular}{|c|c|c|c|c|}
\hline & PA & NGN & BC & OGN \\
\hline Age (years) & $24(20-26.5)$ & $26(22.5-29)$ & $25.5(19.5-31)$ & 33 (no IQR) \\
\hline Sex $(F / M)$ & $6 / 4$ & $5 / 4$ & $6 / 4$ & $2 / 3$ \\
\hline $\mathrm{FEV}_{1}$ (I) & $2.6(2.0-3.2)$ & $2.6(2.1-2.9)$ & $2.5(1.1-3.4)$ & 1.9 (no IQR) \\
\hline $\mathrm{FEV}_{1}(\%$ predicted) & $70.2(65.1-89.7)$ & $75.0(65.1-94.0)$ & $67.2(40.6-86.5)$ & 62.6 (no IQR) \\
\hline C-reactive protein $(\mathrm{mg} / \mathrm{ml})$ & $3.0(1.0-4.0)$ & $4.0(1.5-8.0)$ & $9.0(2.0-15.0)$ & 3.0 (no IQR) \\
\hline Blood neutrophils $\left(\times 10^{9}\right.$ cells/I) & $4.4(3.2-9.7)$ & $4.5(3.8-5.9)$ & $6.2(4.3-9.8)$ & 3.4 (no IQR) \\
\hline White cell count $\left(\times 10^{9}\right.$ cells $\left./ \mathrm{I}\right)$ & $7.3(6.4-9.9)$ & $8.2(7.5-8.8)$ & $8.9(7.4-13.8)$ & 6.7 (no IQR) \\
\hline Total cells $\left(\times 10^{6} / \mathrm{g}\right.$ sputum $)$ & $4.2(3.2-14.4)$ & $5.2(0.7-7.4)$ & $3.6(0.9-12.2)$ & 3.1 (no IQR) \\
\hline
\end{tabular}

sample of CF patients was recruited matched as closely as possible for lung function, age, sex, and genetics (table 1). No power calculation was done before the study. A blood sample was also taken for measurement of C-reactive protein (CRP) concentrations, white cell count, and neutrophil count. Informed written consent was obtained from all patients. The study was approved by the ethics committee of The Queen's University of Belfast and carried out in accordance with the Declaration of Helsinki (1989). Informed written consent was obtained from all patients.

\section{Sputum microbiology}

Patients were categorised as follows into four groups based on their microbiological status over the preceding 12 months and where the patients continued to culture the same organism during the study period as in the previous 12 week period: (1) $P$ aeruginosa (PA) group where patients had been chronically colonised with PA for at least 12 months before commencement of the study; (2) B cenocepacia (BC) group where patients had been chronically colonised with $B$ cenocepacia for at least 12 months before commencement of the study; (3) no Gram negative growth (NGN) where patients had no significant bacteria noted for at least 12 months before commencement of the study; and (4) other Gram negative group (OGN) where patients had been colonised with other Gram negative organisms for at least 12 months before commencement of the study (predominantly Stenotrophomonas maltophilia) and in the absence of PA and $\mathrm{BC}$.

\section{Sputum induction}

Sputum induction was performed using the Sonix 2000 nebuliser (Clement Clarke International Ltd, Harlow, UK), adapting methods described by Pavord et al. ${ }^{16}{ }^{17}$ In brief, 3\% saline was nebulised for 20 minutes. Forced expiratory volume in 1 second $\left(\mathrm{FEV}_{1}\right)$ and forced vital capacity (FVC) (Vitalograph) and their reversibility to salbutamol were determined by spirometry according to ATS standards. Oxygen saturation and pulse rate were recorded throughout (Sat-Trak pulse oximeter; SensorMedics, Bilthoven, The Netherlands). Nebulisation was stopped if $\mathrm{FEV}_{1}$ fell by $>20 \%$ at any stage. If this occurred, salbutamol $(200 \mu \mathrm{g})$ was administered as necessary. Sputum was collected in a sterile plastic pot placed on ice.

\section{Sputum processing}

Sputum samples were processed within 2 hours. The total weight of the sample was recorded. Sputum plugs were selected and processed, adapting methods described by Pavord et al. ${ }^{16}{ }^{17}$ The supernatant was removed from the cell pellet and stored at $-70^{\circ} \mathrm{C}$ for future analysis. Cell viability was determined by the trypan blue exclusion method. Cytospin slides were made and stained with Diff Quik (Clin-Tech Ltd, Essex, UK). Concentrations of interleukin (IL)-6, IL-8 and IL-10 in the supernatant were measured using a quantitative sandwich immunoassay (Pelikine Compact ELISA kits). Neutrophil elastase (NE) was measured using a quantitative continuous (kinetic) assay. ${ }^{18}$ Sputum was cultured for identification of major pathogens using routine microbiological methods as outlined by the United Kingdom Cystic Fibrosis Trust. ${ }^{19}$ C-reactive protein (CRP) was measured by nephelometry.

\section{Flow cytometry}

Cell suspensions $\left(1 \times 10^{6}\right.$ cells $\left./ \mathrm{ml}\right)$ were washed twice with phosphate buffered saline/1\% bovine serum albumin (PBS/ $1 \%$ BSA) and resuspended in the binding buffer supplied with the annexin V-FITC (fluorescein isothiocyanate) (Pharmingen). Cell suspensions were then labelled with annexin V-FITC (AV) and propidium iodide (PI) or antihuman monoclonal CD66b-FITC (BD PharMingen). Sputum cells were gated on a granulocyte population using a forward scatter and side scatter plot. Negative controls consisted of omission of AV and PI, as positive controls are not readily accessible for apoptosis detection. Mouse $\operatorname{IgM} \kappa$ isotype control was used in flow cytometric assessment of CD66b. To eliminate cellular debris from the analysis, the discrimination level was set at 100. Analysis of samples was performed using the Immuno 4 software programme which involved subtraction of negative controls from positive samples. Flow cytometry was performed as previously described. ${ }^{20}$

\section{Morphology}

Cytospin slides were also prepared from sputum and evaluated by light microscopy for the presence of apoptotic cells. In order to evaluate the proportion of apoptotic cells in neutrophils recovered from sputum, at least 500 neutrophils were graded for apoptosis on each cytospin slide. Cells showing typical features of apoptosis such as nuclear chromatin condensation, nuclear coalescence, and cell shrinkage were considered apoptotic. ${ }^{21}$

\section{Statistical analysis}

Results are reported as median values with interquartile range. Data were analysed using a Kruskal-Wallis one-way ANOVA which, if significant, was followed by non-parametric Mann-Whitney two sample (non-matched) tests. A $p$ value of $<0.05$ was considered statistically significant. When the measured mediator concentration was below the detection limit of the assay, a value equal to the detection limit of the assay was substituted in the statistical analysis. Statistical analysis was performed using SPSS Version 11.5 for Windows.

\section{RESULTS}

Thirty four patients with CF completed the study ( 10 with PA infection, 10 with $\mathrm{BC}$ infection, nine NGN, and five OGN); their demographic data are shown in table 1 . There were no significant differences in lung function (\% predicted $\mathrm{p}=0.4$ or litres, $\mathrm{p}=0.8)$ or age $(\mathrm{p}=0.1)$ between any of the groups 

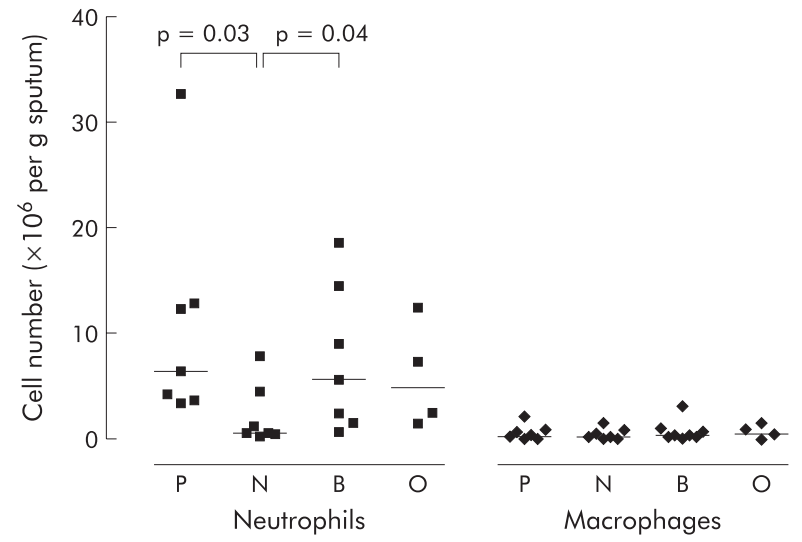

Figure 1 Neutrophil $\left(\times 10^{6} / \mathrm{g}\right.$ sputum) and macrophage $\left(\times 10^{6} / \mathrm{g}\right.$ sputum) cellular data for cystic fibrosis patients colonised with Pseudomonas aeruginosa (P), no Gram negative infection (N), Burkholderia cenocepacia (B), and other Gram negative infections (O). Data are shown as individual points; the horizontal lines represent median values. $p$ values reaching statistical significance are shown.

studied when analysed using a Kruskal-Wallis one way ANOVA.

\section{CRP, blood neutrophils, and white cell counts}

There were no significant differences in CRP levels, blood neutrophils $\left(\times 10^{9}\right.$ cells $\left./ 1\right)$, and total white cell counts between any of the four patient groups $(p=0.21, p=0.439$ and $\mathrm{p}=0.213$, respectively, Kruskal-Wallis test; table 1).

\section{Sputum cell counts}

The total number of cells isolated per gram of sputum did not differ significantly between patients with CF colonised with different micro-organisms $(p=0.8$, Kruskal-Wallis test; table 1$)$. The number of neutrophils $\left(\times 10^{6} / \mathrm{g}\right.$ sputum $)$ in the sputum of patients with PA infection (Kruskal-Wallis $\mathrm{p}=0.05$, median (IQR) $6.3(3.5-12.7), \mathrm{p}=0.03)$ and $\mathrm{BC}$ infection (median (IQR) $5.7 \quad(1.5-14.5), \quad p=0.04)$ was significantly higher than in the NGN group (median (IQR)

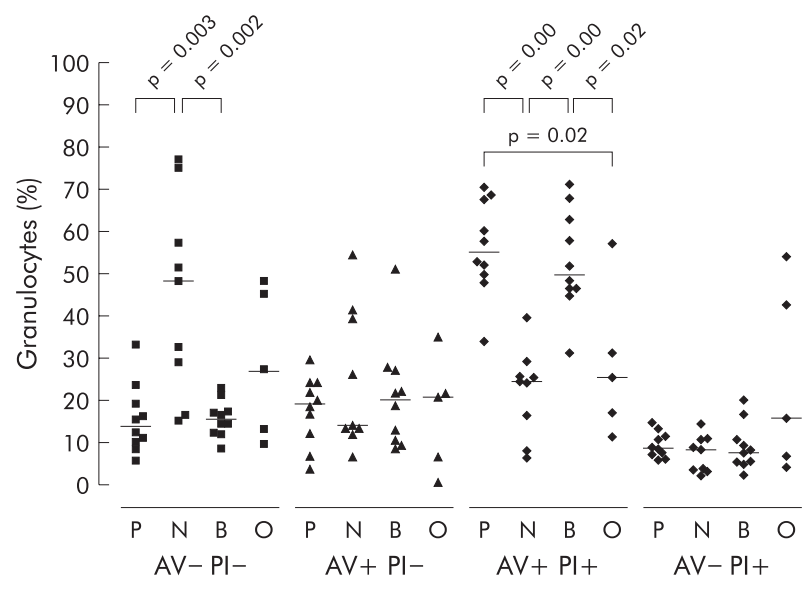

Figure 2 Flow cytometry data for sputum cells labelled with either annexin $\mathrm{V}(\mathrm{AV})$ or propidium iodide (PI) for cystic fibrosis patients colonised with Pseudomonas aeruginosa (P), no Gram negative infection (N), Burkholderia cenocepacia (B), and other Gram negative infections (O). Cells labelled with AV only (AV+/PI-) are apoptotic, cells labelled with $\mathrm{Pl}$ only $(\mathrm{AV}-/ \mathrm{PI}+)$ are necrotic, cells labelled with neither $\mathrm{AV}$ nor $\mathrm{PI}$ (AV-/PI-) are neither apoptotic nor necrotic, and cells labelled with AV and $\mathrm{PI}(\mathrm{AV}+/ \mathrm{Pl}+)$ have secondary necrosis. Data are shown as individual points; the horizontal lines represent median values. $p$ values reaching statistical significance are shown.

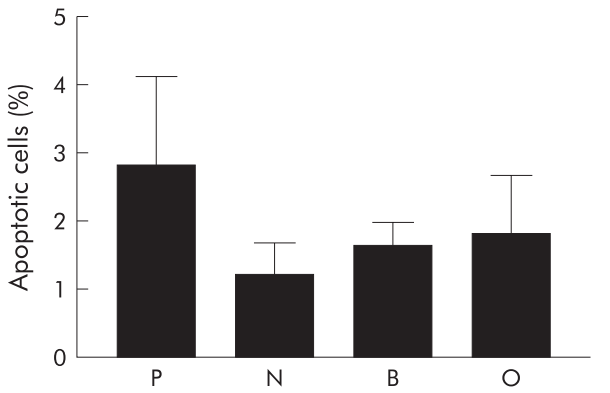

Figure 3 Percentage of apoptotic cells in sputum as assessed by morphology for cystic fibrosis patients colonised with Pseudomonas aeruginosa (P), no Gram negative infection (N), Burkholderia cenocepacia (B), and other Gram negative infections (O). Data are shown as mean (SE).

0.5 (0.5-4.3); fig 1). The number of macrophages $\left(\times 10^{6} / \mathrm{g}\right.$ sputum) did not differ significantly between each of the four groups (Kruskal-Wallis $p=0.9$; fig 1 ). The percentage of macrophages (data not shown) was negatively correlated with the percentage of cells with secondary necrosis $(R=-0.555, \mathrm{p}=0.004)$.

\section{Apoptosis and activation}

There were no significant differences in the percentage of apoptotic $(\mathrm{AV}+\mathrm{PI}-)$ granulocyte numbers as measured by Annexin V staining or morphology in each of the four patient groups studied (Kruskal-Wallis $p=0.8$ and $p=0.4$, respectively; figs 2 and 3). As previously reported, ${ }^{20}$ a lower proportion of apoptotic cells was detected by light microscopy than by flow cytometry. This is probably due to the different stages of apoptosis measured by the two methods. ${ }^{22}{ }^{23}$ There were no significant differences in necrotic (AV-PI+) granulocyte numbers in each of the patient groups studied (Kruskal-Wallis, $\mathrm{p}=0.4$; fig 2). Patients infected with PA (Kruskal-Wallis $\mathrm{p}=0.01$, median (IQR) $14.2 \%$ (9.4-21.6)) or BC (median (IQR) 15.8\% (12.3-19.5)) had a significantly lower percentage of viable $(\mathrm{AV}-\mathrm{PI}-)$ granulocytes in sputum than patients in the NGN group (median (IQR) $48.4 \%$ (23.0-66.4), $\mathrm{p}=0.003$ and $\mathrm{p}=0.002$, respectively). Conversely, patients with PA (Kruskal-Wallis $\mathrm{p}<0.0001$, median (IQR) 55.5\% (49.1-68.5)) or BC (median (IQR) $50.4 \%$ (46.0-65.8)) infection had significantly higher levels of secondary necrotic granulocytes (AV+PI+) in their sputum than patients in the NGN (median (IQR) 24.8\% (12.5-27.8), $\mathrm{p}<0.0001$ and $\mathrm{p}<0.0001$, respectively) or OGN groups

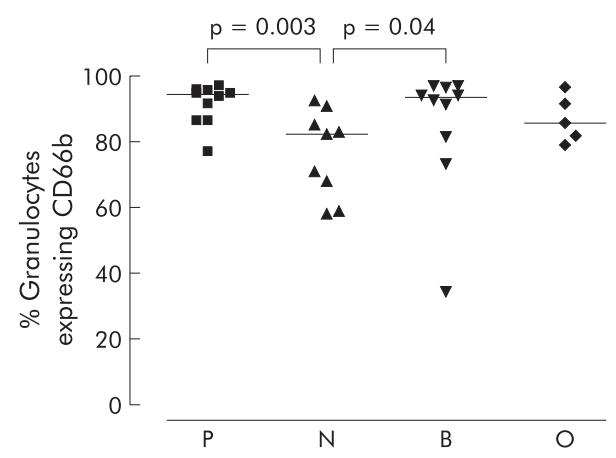

Figure 4 Flow cytometry data for sputum cells labelled with anti-CD66b for cystic fibrosis patients colonised with Pseudomonas aeruginosa (P), no Gram negative infection (N), Burkholderia cenocepacia (B), and other Gram negative infections (O). Data are shown as individual points; the horizontal lines represent median values. p values reaching statistical significance are shown. 


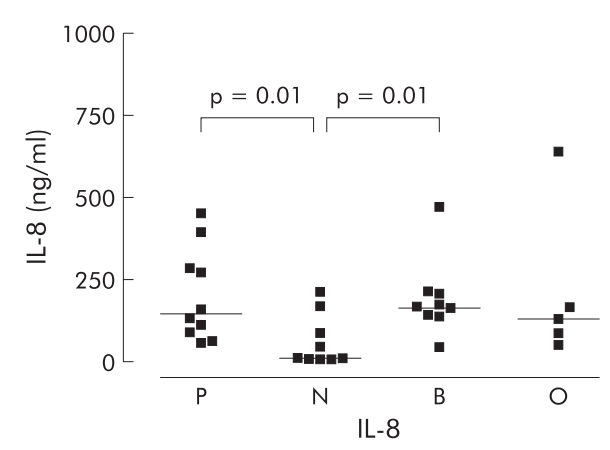

Figure 5 Concentrations of interleukin 8 (IL-8) in sputum sol from cystic fibrosis patients colonised with Pseudomonas aeruginosa (P), no Gram negative infection (N), Burkholderia cenocepacia (B), and other Gram negative infections (O). Data are shown as individual points. Horizontal lines represent the median values. $p$ values reaching statistical significance are shown.

(median 26.0\% (no IQR), $\mathrm{p}=0.02$ and $\mathrm{p}=0.02$, respectively; fig 2).

Sputum neutrophils obtained from all four patient groups were found to be highly activated as indicted by surface expression of the granulocyte activation marker CD66b (fig 4). Sputum neutrophils from patients infected with PA (Kruskal-Wallis $\mathrm{p}=0.04$, median (IQR) 94.2\% (86.4-95.4)) or BC (median (IQR) 93.4\% (77.4-96.8)) had a significantly higher percentage of granulocytes expressing CD66b than patients in the NGN group (median (IQR) $82.1 \%$ (63.3-87.9), $\mathrm{p}=0.003$ and $\mathrm{p}=0.043$, respectively).

\section{Supernatant mediators}

The concentrations of IL-8 $(\mathrm{ng} / \mathrm{ml})$ in sputum sol were significantly higher in patients infected with PA (KruskalWallis, $\mathrm{p}=0.03$, median (IQR) $145.4 \mathrm{ng} / \mathrm{ml}$ (75.3-337.3)) and BC (median (IQR) $166.3 \mathrm{ng} / \mathrm{ml}(7-209.9)$ ) than those in the NGN group (median (IQR) $13.2 \mathrm{ng} / \mathrm{ml}$ (7.3-128.4), $\mathrm{p}=0.01$ and $\mathrm{p}=0.01$; fig 5$)$. There were no significant differences between the groups in levels of IL-6 (pg/ml) or IL$10(\mathrm{pg} / \mathrm{ml})$ (Kruskal-Wallis ANOVA, $\mathrm{p}=0.58$ and $\mathrm{p}=0.12$, respectively; fig 6). Three anomalous points have been omitted from the IL- 6 figure to allow easier comparison of levels between groups; the omissions are as follows: $\mathrm{P}$, 1927.4 ng/ml; N, 3043.09 ng/ml; O, 3366.15 ng/ml. Removal of the anomalous points did not alter the statistical findings. The concentration of $\mathrm{NE}(\mathrm{mg} / \mathrm{ml})$ in sputum sol was significantly increased in patients infected with PA

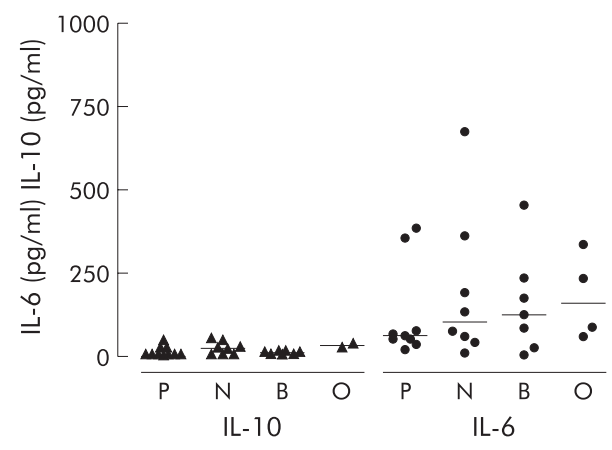

Figure 6 Concentrations of interleukins 6 and 10 (IL-6 and IL-10) in sputum sol from cystic fibrosis patients colonised with Pseudomonas aeruginosa (P), no Gram negative infection (N), Burkholderia cenocepacia (B), and other Gram negative infections (O). Data are shown as individual points. Horizontal lines represent median values. $p$ values reaching statistical significance are shown.
(Kruskal-Wallis, $\mathrm{p}=0.02$, median (IQR) $35.2 \mathrm{mg} / \mathrm{ml}$ (14.496.0)), BC (median (IQR) $62.4 \mathrm{mg} / \mathrm{ml}$ (16.6-96.8)), or OGN (median $49.4 \mathrm{mg} / \mathrm{ml}$, no IQR) compared with the NGN group (median (IQR) $9.6 \mathrm{mg} / \mathrm{ml}$ (3.5-29.2), $\mathrm{p}=0.02, \mathrm{p}=0.01$, $\mathrm{p}=0.03$ respectively; fig 7$)$. Sputum sol levels of IL-8 were positively correlated with NE levels $(R=0.60, \mathrm{p}<0.0001)$.

\section{DISCUSSION}

The main aim of this study was to compare levels of sputum neutrophil apoptosis, necrosis, and activation in four groups of clinically stable CF patients matched as closely as possible for age, sex, and lung function who were colonised with micro-organisms commonly found in the CF lung. A power calculation was not performed before commencement and this is a recognised limitation of this study. We also determined levels of the inflammatory mediators IL-6, IL-8, IL-10, NE, and cell numbers in sputum, our hypothesis being that levels of apoptosis and necrosis may be modulated by the dominant micro-organism colonising the lung and hence influence the inflammatory milieu of the lung. We found significant differences in levels of cell death, cytokine production, and cell counts in sputum between patients infected with Gram negative organisms (PA and BC) and those without Gram negative infection. We did not find significant differences in these inflammatory markers between patients infected with PA and those infected with BC, which suggests that the inflammatory reaction provoked by these two micro-organisms is similar.

This is the first study to compare levels of neutrophil cell death and inflammatory markers in sputum samples from clinically stable patients with CF colonised with different lung pathogens. Levels of necrotic (AV+PI+) neutrophils were very similar between the groups studied, with no significant variations observed. Patients infected with PA or BC had a significantly lower number of viable (AV-PI-) granulocytes in sputum than those with no Gram negative infection. When neutrophils are recruited to a site of infection, their life span is extended by the actions of pro-inflammatory cytokines and death is then induced as a consequence of phagocytosis of microbes and activation of microbicidal mechanisms. ${ }^{24}$ This induction of death as a result of microbe ingestion provides a probable explanation for the lower numbers of viable neutrophils observed in the infected groups. Induction of neutrophil death following phagocytosis may be limited in patients infected with organisms such as PA, which can successfully resist neutrophil phagocytosis by switching from a non-mucoid variant to a mucoid phenotype by forming large exopolysaccharide coated microcolonies after onset of

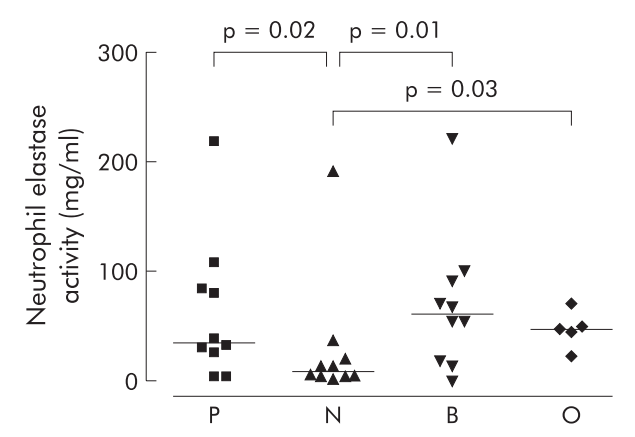

Figure 7 Concentrations of neutrophil elastase in sputum sol from cystic fibrosis patients colonised with Pseudomonas aeruginosa (P), no Gram negative infection (N), Burkholderia cenocepacia (B), and other Gram negative infections (O). Data are shown as individual points. Horizontal lines represent the median values. $p$ values reaching statistical significance are shown. 
infection. ${ }^{25}$ This biofilm formation then results in frustrated neutrophil phagocytosis. ${ }^{25}$

The mean level of apoptosis (AV+PI-) observed in the sputum of patients with CF was around $20 \%$ when analysed by annexin $\mathrm{V}$ staining. This is in agreement with levels observed in CF sputum by Vandivier et al. ${ }^{26}$ There were no significant differences in levels of apoptotic granulocytes between the groups studied. A trend for higher median levels of apoptosis was observed in the PA and BC groups than in the NGN group when apoptosis was assessed by flow cytometry and morphology, although this did not reach statistical significance.

The percentage of secondary necrotic granulocytes was significantly higher in patients colonised with PA and BC than in the NGN group. Secondary necrosis of apoptotic cells is likely to occur if phagocytic clearance is rendered ineffective. Phagocytic clearance may be ineffective for a number of reasons including a lack of mature inflammatory macrophages, overwhelming of the clearance system by massive waves of apoptosis, or failure of phagocytic recognition systems. ${ }^{9}$ If clearance is slower than rates of transition of apoptotic to secondary necrotic, the increase in rates of apoptosis might be translating into the significant increase observed in secondary necrosis. Secondary necrosis of neutrophils exacerbates the inflammatory response and results in further tissue damage. $\mathrm{BC}$ and PA have both been shown to induce neutrophil apoptosis through the production of the toxins hemolysin and pyocyanin, respectively. ${ }^{12}{ }^{13}$ Induction of non-inflammatory apoptosis may prove advantageous to the infecting microbe; however, extensive tissue damage exists in patients infected with PA or BC, which suggests that excessive apoptosis overwhelms phagocytic clearance resulting in secondary necrosis. Levels of pyocyanin or hemolysin were not measured in this study.

Apoptosis is an intrinsic process, however; it can also be modulated by cytokines such as IL- 8 which has been shown to delay spontaneous apoptosis of neutrophils in a dose dependent manner. ${ }^{27}$ Levels of IL-8 were significantly higher in patients infected with PA and BC than in NGN patients, although this was not coupled with significantly lower levels of apoptosis in these patients. However, IL-8 also acts as a major neutrophil chemoattractant and, indeed, increased neutrophil numbers were observed in the PA and BC patient groups. The number of neutrophils $\left(\times 10^{6} / \mathrm{g}\right.$ sputum) was significantly higher in the PA and BC groups than in the NGN group. However, the number of macrophages $\left(\times 10^{6} / \mathrm{g}\right.$ sputum) was not significantly different between the four groups studied. An increase in neutrophil numbers without an accompanying increase in macrophage numbers may have an adverse effect on the efficiency of clearance of apoptotic cells. This may contribute to the significantly higher levels of secondary necrotic cells observed in the PA and BC groups. The total number of cells/g sputum isolated did not differ significantly between any of the CF groups recruited. It is interesting to note that, even though patients with no Gram negative infection remain relatively well, neutrophil infiltration of the lung is still observed at levels comparable with patients infected with major lung pathogens.

As previously mentioned, clearance is a critical feature of the apoptotic process, providing deletion of large numbers of neutrophils while preserving the structure and function of surrounding tissue. ${ }^{28}$ Exposure of phosphatidylserine (PS) on the outer leaflet of the plasma membrane is a well recognised surface change which occurs early in the apoptotic process. ${ }^{29}{ }^{30}$ PS expression coupled with the dominant role of the PS receptor suggests that interaction between the two plays a crucial role in uptake of apoptotic cells. ${ }^{26}{ }^{31}$ Cleavage of the PS receptor by NE specifically disrupts phagocytosis of apoptotic cells. ${ }^{26}$ Our data show significantly increased levels of NE in the sputum sol of patients infected with PA and BC, although we found no correlation with the percentage of secondary necrotic cells present in the sputum. Decreased neutrophil clearance has also been observed as a consequence of the induction of macrophage apoptosis in the presence of tumour necrosis factor $\alpha$ and increased levels of sodium chloride, resulting in conditions of hyperosmolarity. ${ }^{32}$ This may partially explain the persistence of inflammation in CF through impairment of macrophage clearance of neutrophils.

CD66b is a membrane bound glycoprotein involved in neutrophil migration and activation; it is present in the intracellular membrane of different subsets of cytoplasmic granules which, during cellular activation, become mobilised resulting in increased expression on the cell surface. ${ }^{33}$ Kinhult et $a l^{34}$ have demonstrated a marked increase in the expression of CD66b on the cell surface of neutrophils following their migration from the bloodstream to the nasal mucosa. We used CD66b as a marker to assess neutrophil activation in each of the study groups. All of the CF patients studied had high levels of CD66b expression as assessed by flow cytometry. Neutrophils from patients infected with PA or BC expressed significantly higher levels of CD66b, probably as a direct result of the presence of bacterial products in the lung.

Activated neutrophils release large quantities of NE which directly damages the airways through the digestion of elastin and other structural proteins in addition to cleaving opsonins and receptors necessary for phagocytosis. ${ }^{35}$ As a result, opsonophagocytosis and killing of PA and other CF related pathogens such as $S$ aureus and $H$ influenzae has been found to be markedly impaired. ${ }^{25} \mathrm{NE}$ also stimulates the release of proinflammatory cytokines such as IL-8 and IL-6, increased levels of which are seen in the CF lung. ${ }^{36-38}$ Once again the significantly higher levels of IL-8 and NE observed in the PA and $\mathrm{BC}$ groups compared with the NGN group may be related to higher levels of neutrophil activation in these groups, or the higher levels of cytokines and proteases observed may result from secondary necrosis. In contrast to this, the levels of IL-10 measured in all the groups were very low, and this is hypothesised to contribute to the development of chronic airways inflammation. ${ }^{39}$

PA and BC infection produced very similar results in terms of levels of cell death and inflammatory mediator production during clinical stability. This study does not provide any obvious explanation for the rapid deterioration seen in patients with BC. How similar these levels would remain during an infective exacerbation remains to be investigated. The role of the epithelium in driving this deterioration may be important. It has been shown that extracellular products of BC stimulate the respiratory epithelium to produce IL-6, IL- 8 and prostaglandin $E_{2}$, suggesting that the interaction between the respiratory epithelium and secreted bacterial products is important. ${ }^{40}$

The results of this study suggest that cell death pathways in patients infected with PA and BC may be impaired. Whether this occurs as a result of altered apoptotic rate or impaired macrophage clearance requires further investigation. Patients with no Gram negative infection were shown to have high levels of neutrophil infiltration, neutrophil activation, and inflammatory mediators in their induced sputum, indicating that, even in the absence of major lung pathogens, these CF patients have an exaggerated inflammatory response. It remains controversial whether excessive lung inflammation is an intrinsic property of the CFTR defect or whether it is secondary to the unique environment of the CF lung. ${ }^{41}$

A vicious cycle of infection and inflammation occurs in CF which makes it difficult to assess the dynamic sequence of events which is cell death and clearance, leaving a number of 
questions unanswered. Which cells cause more damage to the lung-activated viable cells or necrotic and secondary necrotic cells? Where does the equilibrium between neutrophil apoptosis and macrophage clearance become dysregulated? The use of induced sputum gives the advantage of providing physiologically true samples of the inflammatory milieu of the CF lung; however, it is very difficult to measure the dynamics of cell clearance within these samples. It is therefore problematic to assess whether apoptosis is inhibited or accelerated in patients infected with PA and BC compared with NGN patients or whether phagocytic clearance mechanisms have been impaired.

Inflammation in the CF lung-like other chronic lung diseases-involves a number of pro-inflammatory pathways and an apparent dampening of the anti-inflammatory pathway. In the CF lung, inflammatory markers are apparent even in the absence of major pathogens such as PA and BC. In the presence of infection this response is heightened further, as shown by increased neutrophil activation and higher levels of inflammatory mediators, which suggests that CF neutrophils may have limited capability in restricting their response to infection. These results suggest that cell death and clearance may be altered in patients with CF who are colonised with PA and $\mathrm{BC}$ compared with those with no Gram negative infection.

\section{ACKNOWLEDGEMENTS}

The authors thank Dr L Martin, School of Pharmacy, The Queen's University of Belfast, for supplying the protease inhibitor Z-Phediphenylphosphonate and Ms K Moffit, School of Pharmacy, The Queen's University of Belfast for measurement of neutrophil elastase.

\section{Authors' affiliations}

A P Watt, J Courtney, M Ennis, J S Elborn, Respiratory Research Group, The Queen's University of Belfast, Belfast, UK

J Moore, Department of Bacteriology, Belfast City Hospital, Belfast, UK

This study was funded by an Ireland-Northern Ireland Co-operation Research Project Grant from the Research \& Development Office, Northern Ireland Health \& Social Services Central Services Agency.

There are no competing interests to declare.

\section{REFERENCES}

1 Tomlin KL, Coll OP, Ceri H. Interspecies biofilms of Pseudomonas aeruginosa and Burkholderia cepacia. Can J Microbiol 2001;47:949-54.

2 Lyczak JB, Cannon CL, Pier GB. Lung infections associated with cystic fibrosis. Clin Microbiol Rev 2002;15:194-222.

3 Frangolias DD, Mahenthiralingam E, Rae S, et al. Burkholderia cepacia in cystic fibrosis. Variable disease course. Am J Respir Crit Care Med 1999; 160:1572-7.

4 Nelson JW, Butler SL, Krieg D, et al. Virulence factors of Burkholderia cepacia. FEMS Immunol Med Microbiol 1994:8:89-97.

5 Straus DC, Lonon MK, Woods DE, et al. Production of an extracellular toxic complex by various strains of Pseudomonas cepacia. J Med Microbiol 1989;30:17-22.

6 Stover GB, Drake DR, Montie TC. Virulence of different Pseudomonas species in a burned mouse model: tissue colonization by Pseudomonas cepacia. Infect Immun 1983;41:1099-104.

7 Hammerle AF, Tatschl C, Jatzko S, et al. The lung as an inflammatory organ Acta Anaesthesiol Scand Suppl 1997;1 11:59-64.

8 Henson PM. Possible roles for apoptosis and apoptotic cell recognition in inflammation and fibrosis. Am J Respir Cell Mol Biol 2003;29(3 Suppl):S70-6.

9 Haslett C. Granulocyte apoptosis and its role in the resolution and control of lung inflammation. Am J Respir Crit Care Med 1999;160:S5-11.

10 Lee A, Whyte MK, Haslett C. Inhibition of apoptosis and prolongation of neutrophil functional longevity by inflammatory mediators. J Leukoc Biol 1993;54:283-8.

11 Zychlinsky A, Sansonetti PJ. Apoptosis as a proinflammatory event: what can we learn from bacteria-induced cell death? Trends Microbiol 1997;5:201-4
12 Usher LR, Lawson RA, Geary l, et al. Induction of neutrophil apoptosis by the Pseudomonas aeruginosa exotoxin pyocyanin: a potential mechanism of persistent infection. J Immunol 2002;168:1861-8.

13 Hutchison ML, Poxton IR, Govan JR. Burkholderia cepacia produces a hemolysin that is capable of inducing apoptosis and degranulation of mammalian phagocytes. Infect Immun 1998;66:2033-9.

14 Dacheux D, Toussaint B, Richard M, et al. Pseudomonas aeruginosa cystic fibrosis isolates induce rapid, type III secretion-dependent, but ExoUindependent, oncosis of macrophages and polymorphonuclear neutrophils. Infect Immun 2000;68:2916-24.

15 Hendry J, Elborn JS, Nixon L, et al. Cystic fibrosis: inflammatory response to infection with Burkholderia cepacia and Pseudomonas aeruginosa. Eur Respir J 1999;14:435-8.

16 Pavord ID, Pizzichini MM, Pizzichini E, et al. The use of induced sputum to investigate airway inflammation. Thorax 1997;52:498-501.

17 Kelly MG, Brown V, Martin SL, et al. Comparison of sputum induction using high-output and low-output ultrasonic nebulizers in normal subjects and patients with COPD. Chest 2002; 122:955-9.

18 Castillo MJ, Nakajima K, Zimmerman M, et al. Sensitive substrates for human leukocyte and porcine pancreatic elastase: a study of the merits of various chromophoric and fluorogenic leaving groups in assays for serine proteases. Anal Biochem 1979;99:53-64.

19 United Kingdom Cystic Fibrosis Trust. Antibiotic treatment for cystic fibrosis. Bromley, Kent, 2000.

20 Watt AP, Brown V, Courtney J, et al. Neutrophil apoptosis, proinflammatory mediators and cell counts in bronchiectasis. Thorax 2004;59:231-6.

21 Zhang X, Moilanen E, Kankaanranta H. Beclomethasone, budesonide and fluticasone propionate inhibit human neutrophil apoptosis. Eur J Pharmacol 2001:431:365-71.

22 Chilvers ER, Cadwallader KA, Reed BJ, et al. The function and fate of neutrophils at the inflamed site: prospects for therapeutic intervention. J R Coll Physicians Lond 2000;34:68-74.

23 Willingham MC. Cytochemical methods for the detection of apoptosis. $J$ Histochem Cytol 1999;47:1101-9.

24 Steensma DP, Timm M, Witzig TE. Flow cytometric methods for detection and quantification of apoptosis. Methods Mol Med 2003;85:323-32.

25 Doring G. Cystic fibrosis respiratory infections: interactions between bacteria and host defence. Monaldi Arch Chest Dis 1997;52:363-6.

26 Vandivier RW, Fadok VA, Hoffmann PR, et al. Elastase-mediated phosphatidylserine receptor cleavage impairs apoptotic cell clearance in cystic fibrosis and bronchiectasis. J Clin Invest 2002;109:661-70.

27 Kettritz R, Gaido ML, Haller H, et al. Interleukin-8 delays spontaneous and tumor necrosis factor-alpha-mediated apoptosis of human neutrophils. Kidney Int 1998;53:84-91.

28 Fadok VA. Clearance: the last and often forgotten stage of apoptosis. J Mammary Gland Biol Neoplasia 1999;4:203-11.

29 Martin SJ, Reutelingsperger CP, McGahon AJ, et al. Early redistribution of plasma membrane phosphatidylserine is a general feature of apoptosis regardless of the initiating stimulus: inhibition by overexpression of $\mathrm{Bcl}-2$ and Abl. J Exp Med 1995;182:1545-56.

30 Verhoven B, Schlegel RA, Williamson P. Mechanisms of phosphatidylserine exposure, a phagocyte recognition signal, on apoptotic T lymphocytes. J Exp Med 1995; 182:1597-601.

31 Fadok VA, Bratton DL, Rose DM, et al. A receptor for phosphatidylserinespecific clearance of apoptotic cells. Nature 2000;405:85-90.

32 Kerby GS, Cottin V, Accurso FJ, et al. Impairment of macrophage survival by $\mathrm{NaCl}$ : implications for early pulmonary inflammation in cystic fibrosis. Am J Physiol Lung Cell Mol Physiol 2002;283:L188-97.

33 Skubitz KM, Campbell KD, Skubitz AP. CD66a, CD66b, CD66c, and CD66d each independently stimulate neutrophils. Leukoc Biol 1996;60:106-17.

34 Kinhult J, Egesten A, Benson M, et al. Increased expression of surface activation markers on neutrophils following migration into the nasal lumen. Clin Exp Allergy 2003;33:1141-6.

35 Konstan MW, Berger M. Current understanding of the inflammatory process in cystic fibrosis: onset and etiology. Pediatr Pulmonol 1997;24:137-42.

36 Bonfield TL, Panuska JR, Konstan MW, et al. Inflammatory cytokines in cystic fibrosis lungs. Am J Respir Crit Care Med 1995;152:2111-8.

37 Bonfield TL, Konstan MW, Berger M. Altered respiratory epithelial cell cytokine production in cystic fibrosis. J Allergy Clin Immunol 1999;104:72-8.

38 Pukhalsky AL, Kapranov NI, Kalashnikova EA, et al. Inflammatory markers in cystic fibrosis patients with lung Pseudomonas aeruginosa infection. Mediators Inflamm 1999;8:159-67.

39 Takanashi S, Hasegawa Y, Kanehira Y, et al. Interleukin-10 level in sputum is reduced in bronchial asthma, COPD and in smokers. Eur Respir J 1999; 14:309-14

40 Fink J, Steer JH, Joyce DA, et al. Pro-inflammatory effects of Burkholderia cepacia on cystic fibrosis respiratory epithelium. FEMS Immunol Med Microbiol 2003;38:273-82.

41 Becker MN, Saver MS, Muhlebach MS, et al. Cytokine secretion by cystic fibrosis airway epithelial cells. Am J Respir Crit Care Med 2004; 169:645-53. 